\title{
A COMPARISON OF REGIONAL FORECASTING TECHNIQUES
}

\author{
John E. Connaughton and Ronald A. Madsen*
}

\section{Introduction}

The need for regional econometric forecasting models and the uses of their results has increased in recent years. A major reason for the growing interest in these models is the increasing recognition that regional economies vary in structure and performance, particularly in their cyclical behavior. Additionally, econometric models may be important for understanding differences in the trend behavior of regional economies.

A major problem that has affected the development of regional models is the substantial cost associated with constructing and maintaining a large scale, well-detailed econometric model. Because of the high cost of these large-scale econometric models, much of the work in regional forecasting has utilized less expensive and less exacting approaches. These methods include both indicator forecasting and autoregressive integrated moving average (ARIMA) methods. These models trade-off linkage and structural information for reduced data needs. More recently there has been an increase in the use of vector autoregressive (VAR) models, which provide limited information about linkages and have modest data requirements, but require substantially greater computing costs.

The purpose of this paper is to compare the quality of forecasts made using three different forecasting methods for a disaggregated regional economy. The procedures produced a set of out-of-sample forecasts for each of ten employment sectors of a regional economy between 1983 and 1988. Forecasting accuracy was measured using mean absolute percent error (MAPE) and mean squared error (MSE) for the altemative forecasting approaches. The first forecasting method employed ordinary least-squares regression (OLSQ). The second method produced forecasts for the ten sectors with ARIMA models, and the third method utilized VAR models to produce the forecasts. The forecast horizon was set at three time periods. This time period selection eliminates the need to forecast values for contemporaneous right-hand side exogenous variables in

\footnotetext{
*Associate Professor and Professor of Economics, University of North Carolina at Charlotte. The authors would like to thank Priyan Attygalle of the Center for Business and Economic Research at UNC-Charlotte and three anonymous referees for their valuable comments.
}

the OLSQ equation. This problem has historically constituted a major source of forecasting error for OLSQ specifications. The comparisons of forecasting accuracy for a regional economy over the seventy-two month period provides an extended evaluation of these methods under widely varying economic conditions.

The OLSQ approach uses a causality based structural model with both endogenous and exogenous explanitory variables to forecast future values of the dependent variable series. The ARIMA approach relies on the information contained in a single variable time series to forecast future values of that series by integrating an autocorrelation pattern and a moving average pattern contained within the series. The VAR approach uses the information contained in a set of time series variables to estimate the future values of that set of variables.

The results of this study should provide valuable insight to regional model builders concerning the general accuracy of alternative regional forecasting techniques. The results should extend the current literature by allowing a comparison of forecasting accuracy for a set of disaggregated employment forecasts over an extended, six year, period of time. This comparison is likely to be of greatest significance to model builders who are making choices on modeling alternatives and do not have the time or resources to fully explore the potential accuracy of each method.

The literature currently provides very limited comparisons of the forecasting accuracy of structural equation, ARIMA, and VAR models at the regional level. Kinal and Ratner (1986) utilize monthly data for a regional economy (the state of New York) to compare the forecasting accuracy of several models. These comparisons included ARIMA and VAR forecasts on four regional variables (Retail Sales, Total Non-Agricultural Employment, Consumer Price Index, and Factory Output) over a four year period. Their results on forecasting accuracy of ARIMA versus VAR varied by year and by forecast variable. Hoehn (1984) concluded that there is no evidence that VAR's can provide more efficient regional forecasts than uninvariate methods, and that there is no clear evidence that structural econometric models of regions can provide efficient forecasts in a systematic way. These papers extended the seminal work done by Anderson (1979) that suggested the promise of VAR in regional forecasting. 
There is a wider body of literature comparing the forecasting accuracy of different methods at the national level dealing with macroeconomic variables. Dhrymes and Peristioni (1988) provide a comparison of forecasting accuracy for a large scale econometric model (the Wharton Mark III model) versus ARIMA covering twenty-three national macrovariables. Their conclusion is that the large scale econometric model provided generally superior forecasting accuracy versus ARIMA forecasts. The strength of this conclusion may be limited by the narrow (1973-75) forecast period. Bagshaw (1986) found that for two widely studied series, advertising and sales, ARIMA and multiple ARIMA models provide better forecasts than do VAR and bayesian VAR models. In a study dealing with selection of lag lengths for VAR models Webb (1985) also produced a comparison of VAR and ARIMA models for three variables (interest rates, real GNP, and the implicit price deflator) with results that differed by variable and forecast horizon. In general the VAR models produced slightly more accurate forecasts than the ARIMA models.

Clearly there is a need for the continued exploration of the relative accuracy of forecasts produced by alternative methods, especially at the regional level. The remainder of this paper provides such an exploration.

\section{The Regional Economy}

The regional economy used to study the accuracy of the altemative forecasting methods was the non-agricultural North Carolina state economy between 1972 and 1988. Monthly employment for each of ten sectors and the monthly state total (non-agricultural) employment were modeled. Non-agricultural employment accounts for more than 98 percent of total employment in North Carolina. North Carolina is the tenth most populous state in the nation and has an economy with a diverse structure, moderate cyclical swings, and strong secular growth. The ten sectors included: 1) mining; 2) construction; 3) durable goods manufacturing; 4) non-durable goods manufacturing; 5) transportation communications, and public utilities (TCPU); 6) wholesale trade; 7) retail trade; 8) finance, insurance, and real estate (FIRE); 9) services; and 10) government. Additionally, for each of the three forecasting methods a total state employment forecast was made by summing the employment forecasts made for each of the ten sectors. The employment data was obtained from the BLS Establishment Series.

OLSQ: The monthly employment data for each sector between 1983-1988 was forecast for each of the ten sectors with OLSQ models based on 1972-1982 data. The exact specification and the beginning forecast equations are presented in Appendix A. Data problems forced the equation for the government sector to be estimated on the basis of 1974-1982 monthly data only. The right-hand variables in each equation were selected from among a group of variables with well established structural links to employment behavior. In all cases the specification providing the minimal mean square error was used, although OLSQ models are considered structural, forecasting accuracy was deemed most important in the selection of the independent variables used in each equation. The forecasting procedure was conducted in two stages. First, employment was forecast for each of the three months of the initial forecast period (the first quarter of 1983). Then, based on historical data (including historical data for the months just forecast), the regression equation was reestimated, and another forecast was made three months forward. Thus, between January 1983 and December 1988 there were 72 forecasts made for each of the ten sectors. The sum of the monthly sector forecasts produced a monthly total employment forecast. Mean absolute percent error (MAPE) and mean squared error (MSE) were calculated for each sector and for the state total by comparing the monthly forecasts with historical employment data. The MAPE and MSE results are reported later in Tables One and Three.

ARIMA: After the data were made stationary, the ARIMA models for each of the ten sectors were specified for the period 1972-1982 (1974-1982 for the government sector) based on the minimal MSE criterion. Specifications of the ARIMA models for each sector are provided in Appendix B. Again, the procedure was to forecast forward three months starting in January 1983 and then, using historical data, re-estimate the models and forecast forward another three months. This procedure produced 72 forecasts for each sector. Sector totals were summed to produce 72 total employment forecasts. The MAPE and MSE results are reported in Tables One and Three.

VAR: The VAR models were constructed for each of the ten sectors based on a one to three period lag and the inclusion of all ten sectors' employment levels. Thus, each sector equation contained thirty right-hand variables. While this choice was essentially arbitrary, Webb (1985) has asserted that there is no clear criterion for selecting lag lengthsand the gains in forecasting accuracy from restricting the parameterization of VAR models are not expected to be large. Since the data base (1972-1982) still provides 102 degrees of freedom ( 88 for the government sector), the simpler specification of the VAR model was adopted.

Similar to the OLSQ and ARIMA forecasts, the VAR procedure also consisted of forecasts made three months forward, with a modification. Since the VAR forecast for the second month in each group of three months required the sector employment levels for the previous month, the 
month were used. Similarly, for the third month forecast, the out-of-sample employment forecasts for the previous two months were used. Thus, the VAR model was dynamic in this sense: for each group of three monthly forecasts, two of the months were partially driven by current forecasts. At the end of each three month period, the historical sector employment levels were brought into the model to replace the out-of-sample forecast values used. Again the VAR models produced 72 monthly forecasts and the sector forecasts were summed to produce 72 monthly state total employment forecasts. Average MAPE and MSE results for the VAR model are presented in Tables One and Three with the results for the OLSQ and ARIMA forecasts.

\section{Results}

The MAPE results presented in the table above are striking. In general, all three approaches produce a uniformly high level of forecasting accuracy.

The overall accuracy of the results is encouraging given the range of performance experienced by the North Carolina economy during this six year period. It is interesting to note that for all three methods the average MAPE for N.C. total employment is lower than for any individual sector's average MAPE. Not surprisingly, this is due to offsetting forecasting errors between sectors for each method. This result does clearly indicate the potential importance of disaggregation when forecasting employment for a specific sector.
The VAR results, with sector average MAPE values that range from 0.29 percent in non-durables manufacturing to 1.35 percent in construction are consistently better than the OLSQ and ARIMA results. The VAR forecasts produced lower average MAPE values than the OLSQ for nine of ten sectors and a lower state total average MAPE. The VAR forecasts produced a lower average MAPE than the ARIMA forecasts in all ten sectors and a lower state total average MAPE.

There are few pronounced differences between the OLSQ and ARIMA forecasts over the 72 month forecasting period. The ARIMA forecasts produced a lower average MAPE for five of the ten sectors and the ARIMA procedure produced a slightly higher average MAPE $(0.45 \%$ versus $0.42 \%$ ) for the state total employment forecast.

As indicated in Table Two, the difference in the VAR average forecasting error for the N.C. Total versus either the corresponding OLSQ or ARIMA forecasts is significant at the .05 level. Additionally, the differences in the average forecasting error by sector for the VAR versus the OLSQ models were significant at the .05 level in seven of ten sectors. In each case, the VAR forecasts were better. The average differences in forecast errors for the VAR models versus the ARIMA models were significant (at the .05 level) in six of ten sectors. In each case the VAR forecasts were better. One of the differences in average forecast error for the OLSQ versus ARIMA models was significant at the .05 level. The ARIMA forecasts for durable goods were significantly better than the OLSQ forecasts, keeping in mind the VAR forecasts for durable

Table 1

Average Mean Absolute Percent Error (MAPE) for North Carolina Monthly Employment Forecasts 1983-1988

\begin{tabular}{llll}
\hline \hline SECTOR & OLSQ & ARIMA & VAR \\
\hline Mining & $1.48 \%$ & $1.45 \%$ & $1.14 \%$ \\
Construction & 1.98 & 1.78 & 1.35 \\
Durables & 1.13 & 0.60 & 0.35 \\
Non-Durables & 0.64 & 0.51 & 0.29 \\
TCPUa & 0.82 & 0.96 & 0.85 \\
Wholesale Trade & 0.92 & 0.96 & 0.54 \\
Retail Trade & 0.80 & 0.83 & 0.62 \\
FIREb & 0.53 & 0.60 & 0.50 \\
Services & 0.93 & 0.95 & 0.47 \\
Government & 0.71 & 0.70 & 0.32 \\
N.C. TOTAL & $0.42 \%$ & $0.45 \%$ & $0.20 \%$ \\
\hline \hline
\end{tabular}

aTransportation, Communication, and Public Utilities - TCPU

bFinance, Insurance, and Real Estate - FIRE 
Table 2

Tests of the Significanne of the Difference in Average Mape Between Methods

\begin{tabular}{lccc}
\hline \hline SECTOR & ARIMA vs OLSQ & VAR vs ARIMA & VAR vs ARIMA \\
\hline Mining & $0.1334 \mathrm{c}$ & 0.6223 & 1.6894 \\
Construction & 0.9572 & $2.2179^{*}$ & 1.5359 \\
Durables & $3.1051^{*}$ & $407526^{*}$ & $2.9477^{*}$ \\
Non-Durables & 1.5683 & $4.9525^{*}$ & $4.0280^{*}$ \\
TCPUa & 0.9556 & 0.1960 & 0.6939 \\
Wholesale Trade & 0.2519 & $2.8730^{*}$ & $3.3054^{*}$ \\
Retail Trade & 0.2101 & $2.4748^{*}$ & $2.2359^{*}$ \\
FIREb & 0.8386 & 0.4469 & 1.2337 \\
Services & 0.1366 & $3.7004^{*}$ & $3.8449^{*}$ \\
Government & 0.1514 & $4.6122^{*}$ & $5.2446^{*}$ \\
N.C. TOTAL & 0.5440 & $2.1141^{*}$ & $5.3047^{*}$ \\
\hline
\end{tabular}

aTransportation, Communication, and Public Utilities - TCPU

bFinance, Insurance, and Real Estate - FIRE

CThe absolute values of $t$ are reported with asterisks $\left(^{*}\right)$ on $t$-values that indicate significant differences at the .05 level (two-tailed tests).

goods was significantly better than both OLSQ and ARIMA forecasts.

Table Three presents the average forecasting results of the three methods in terms of MSE similar to the way the average MAPE results were reported. Table Four presents the tests of the significance of the differences in average MSE for each method versus the other methods.
The results presented in Table Three indicate that based on average MSE, the VAR forecasts are generally better than either the OLSQ or ARIMA forecasts. The VAR forecasts were, on average, better than the OLSQ forecasts in eight of ten sectors and for the North Carolina total employment forecast. The VAR forecasts were, on average, better than the ARIMA forecasts in nine of ten

Table 3

Average Mean Squared Error (MSE) for North Carolina Monthly Employment Forecasts for 1983-1988AVERAGE

\begin{tabular}{lrrr}
\hline \hline SECTOR & OLSQ & ARIMA & VAR \\
\hline Mining & 0.009 & 0.008 & 0.005 \\
Construction & 10.869 & 9.009 & 13.893 \\
Durables & 33.970 & 7.637 & 3.355 \\
Non-Durables & 18.237 & 10.830 & 3.012 \\
TCPUa & 2.061 & 2.935 & 2.763 \\
Wholesale Trade & 3.760 & 3.783 & 1.191 \\
Retail Trade & 20.399 & 25.674 & 10.437 \\
FIREb & 0.616 & 0.787 & 0.595 \\
Services & 29.996 & 30.427 & 16.328 \\
Government & 18.712 & 14.947 & 2.701 \\
N.C. TOTAL & 232.630 & 259.970 & 38.348 \\
\hline
\end{tabular}

aTransportation, Communication, and Public Utilities - TCPU

bFinance, Insurance, and Real Estate - FIRE 
sectors and for the North Carolina total employment forecast. As shown in Table Four most of these differences are statistically significant.

The OLSQ forecasts were better than the ARIMA forecasts for five of ten sectors. As indicated in Table Four, none of these differences were statistically significant. The ARIMA forecasts were, likewise, better than OLSQ forecasts for five of ten sectors and two of these five differences were statistically significant as shown in Table Four. The OLSQ forecast for North Carolina total employment was better than the ARIMA forecasts, as measured by average MSE, but the difference was not significant. The differences in the magnitudes of the average MSE by sector for each forecasting method reflects both differences in the level of employment by sector and differences in forecasting accuracy by sector. Differences in forecasting accuracy by sector were more clearly identifiable in the average MAPE results reviewed earlier.

The overall results indicate that for sector specific monthly employment forecasts over the 72 month period, 1983-1988, the VAR method yielded results more accurate than the OLSQ or ARIMA results. It can be argued the short-term nature of the forecasts play to the strength of VAR forecasting methods. However, it should be kept in mind that longer term forecasts with the OLSQ model would present the added difficulty of first forecasting the right-hand variables which, in turn, would lead to increased forecasting errors not related to the method itself.

\section{Conclusions}

This study was initiated to provide a comparison of the forecasting accuracy of three alternative forecasting methods; OLSQ, ARIMA, and VAR. These methods were used to forecast a set of disaggregated regional economic variables, monthly employment by industry sector, over an extended period of time (1983-1988). The results indicated that all three methods produced, on average, accurate forecasts as measured by the mean absolute percent error (MAPE) and the mean squared error (MSE). The variability in the magnitude of the MAPE or MSE for each method suggests the importance of forecasting at a level of disaggregation appropriate to the intended use of the forecasts.

The general pattern of comparative results is reasonably clear using either MAPE or MSE as a measure of accuracy. The VAR forecasts were as good or better than the OLSQ and ARIMA forecasts for each of the ten sectors studied and for the total employment forecasts. The differences between the OLSQ and ARIMA forecasts were small and, in general, not significant. For the variables studied and the time periods for which forecasts were made, the VAR method provided better results.

The results of this study do not establish the unequivocal superiority of VAR methods over OLSQ or ARIMA methods in regional forecasting. They do support other research that suggests VAR forecasting methods

Table 4

Tests of the Significance of the Difference in Average MSE Between Methods

\begin{tabular}{lccc}
\hline SECTOR & ARIMA vs OLSQ & VAR vs OLSQ & VAR vs ARIMA \\
\hline Mining & $0.3603^{c}$ & $2.4126^{*}$ & $2.4546^{*}$ \\
Construction & $3.9071^{*}$ & 0.4632 & 0.7395 \\
Durables & $1.9999^{*}$ & $2.3424^{*}$ & 1.8876 \\
Non-Durables & 1.2445 & $2.7381^{*}$ & $3.5934^{*}$ \\
TCPUa & 1.0761 & 0.7202 & 0.1626 \\
Wholesale Trade & 0.0181 & $2.4918^{*}$ & $2.8299^{*}$ \\
Retail Trade & 0.7901 & $2.7117^{*}$ & $2.6161^{*}$ \\
FIREb & 0.9723 & 0.1281 & 0.9918 \\
Services & 0.0574 & 0.4197 & 1.5957 \\
Government & 0.7207 & $3.8370^{*}$ & $3.8432^{*}$ \\
N.C. TOTAL & 0.4023 & $3.8336^{*}$ & $4.8495^{*}$ \\
\hline \hline
\end{tabular}

aTransportation, Communication, and Public Utilities - TCPU

bFinance, Insurance, and Real Estate - FIRE

CThe absolute values of $t$ are reported with asterisks $\left(^{*}\right)$ on $t$-values that indicate significant differences at the .05 level (two-tailed tests). 
offer a potentially useful alternative to structural equation models and ARIMA models. Simple VAR specification forecasts do appear to offer an easily-obtained forecasting accuracy benchmark that can be used to evaluate the forecasting accuracy of structural equation and other types of forecasting models. Additionally, the results clearly indicate the importance of disaggregating regional forecasts regardless of the forecast method chosen. 


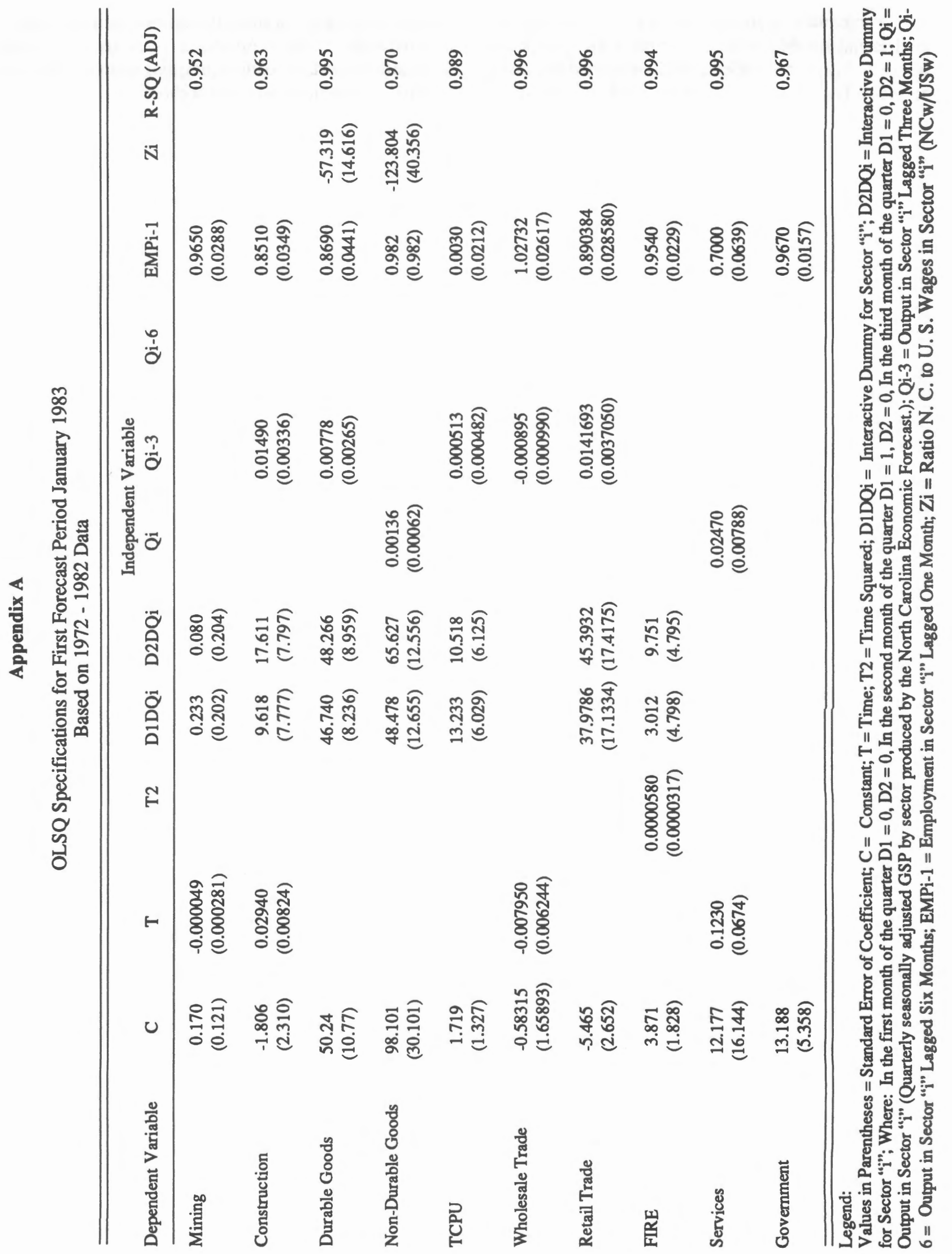




\section{Appendix B}

Specification of ARIMA Models by Sector

$\begin{array}{ll}\text { Mining } & 212 \\ \text { Construction } & 313 \\ \text { Durables } & 313 \\ \text { Non-Durables } & 212 \\ \text { TCPU } & 310 \\ \text { Wholesale Trade } & 211 \\ \text { Retail Trade } & 211 \\ \text { FIRE } & 313 \\ \text { Services } & 313 \\ \text { Government } & 212\end{array}$

Note: The first number in the above specifications is the autoregression order, the second number indicates the extent of differencing, and the third number is the moving average order.

\section{References}

Anderson, P.A., "Help for the Regional Economic Forecaster: Vector Autoregression," Federal Reserve Bank of Minneapolis Ouarterly Review, 3, Summer (1979), 2-7.

Bagshaw, M.L. "Comparison of Univariate Arima, Multivariate Arima, and Vector Autoregressive Forecasting," Working Paper 8602, Federal Reserve Bank of Cleveland, May 1986. Dhrymes, P.J., and S.C. Peristiani, "A Comparison of Forecasting Performance of WEFA and ARIMA Time Series Methods," International Journal of Forecasting,4, no. 1 (1988) 81-101.

Hoehn, J.G. “A Regional Economic Forecasting Procedure
Applied to Texas," Working Paper 8402, Federal Reserve Bank of Cleveland, September 1984.

Kinal, T. and J. Ratner, "A VAR Forecasting Model of a Regional Economy: Its Construction and Comparative Accuracy," International Regional Science Review, 10, no. 2 (1986), 113-26.

Roberds, W. And R.M. Todd, "Forecasting and Modeling the U.S. Economy in 1986-88," Federal Reserve Bank of Minneapolis Quarterly Review, II, Winter (1987), 7-20.

Webb, R.H. "Tow ard More Accurate Macroeconomic Forecasts from Vector Autoregression," paper presented at the Westem Economic Association Conference, Anaheim, California, July 1985, 1-17. 